\title{
A!
}

This is an electronic reprint of the original article.

This reprint may differ from the original in pagination and typographic detail.

Ylä-Oijala, Pasi; Tzarouchis, Dimitrios; Wallén, Henrik; Sihvola, Ari

\section{Spurious-Free Surface Integral Equation Characteristic Mode Formulation for Dielectric Bodies}

Published in:

2018 IEEE AP-S Symposium on Antennas and Propagation and URSI CNC/USNC Joint Meeting (AP-S/URSI

2018) Proceedings, July 8 - 13, 2018, Boston, Massachusetts, U.S.A

DOI:

10.1109/APUSNCURSINRSM.2018.8608430

Published: 01/01/2018

Document Version

Peer reviewed version

Please cite the original version:

Ylä-Oijala, P., Tzarouchis, D., Wallén, H., \& Sihvola, A. (2018). Spurious-Free Surface Integral Equation Characteristic Mode Formulation for Dielectric Bodies. In 2018 IEEE AP-S Symposium on Antennas and Propagation and URSI CNC/USNC Joint Meeting (AP-S/URSI 2018) Proceedings, July 8 - 13, 2018, Boston, Massachusetts, U.S.A (pp. 2453-2454). (Digest of the IEEE Antennas and Propagation Society International Symposium). IEEE. https://doi.org/10.1109/APUSNCURSINRSM.2018.8608430

This material is protected by copyright and other intellectual property rights, and duplication or sale of all or part of any of the repository collections is not permitted, except that material may be duplicated by you for your research use or educational purposes in electronic or print form. You must obtain permission for any other use. Electronic or print copies may not be offered, whether for sale or otherwise to anyone who is not an authorised user. 


\title{
Spurious-Free Surface Integral Equation Characteristic Mode Formulation for Dielectric Bodies
}

\author{
Pasi Ylä-Oijala, Dimitrios C. Tzarouchis, Henrik Wallén and Ari Sihvola \\ Department of Electronics and Nanoengineering \\ Aalto University, Espoo, Finland, pasi.yla-oijala@ aalto.fi
}

\begin{abstract}
A new surface integral equation based formulation for computing characteristic modes of lossless and lossy dielectric and magneto-dielectric bodies is presented. For lossy objects, the imaginary part of an eigenvalue gives the ratio of the dissipated and radiated power, while the real part is connected to the ratio of the reactive and radiated power.
\end{abstract}

\section{INTRODUCTION}

The theory of characteristic modes (TCM) has recently attained a lot of interest in the antenna community. TCM provides excitation-independent eigensolutions that depend only on the shape and material of the structure. This allows analysis of the fundamental scattering and radiation properties of arbitrarily shaped structures.

The theoretical background of the TCM for PEC structures has been established [1], and refined for the electric field integral operator (EFIO) [2], already in the 1970's. For the magnetic and combined field integral operators (MFIO, CFIO), TCM has been developed only very recently [5]. The particularly important outcome of [5] is that, in order to obtain the same eigenvalues and eigencurrents as with the EFIO-based TCM, the roles of the real and imaginary parts of the MFIO appearing in the eigenvalue equation should be swapped.

For penetrable bodies the situation is more complicated, since the surface integral equation formulations typically include both the interior and exterior integral operators, and both electric and magnetic fields and currents. In [3] TCM was formulated for the symmetrical PMCHWT equations. This formulation, however, has been found to produce spurious modes [4]. Several alternative TCM formulations for dielectric bodies have been proposed [7], [8], [9], [10]. All these formulations, however, still either couple the internal and external equations and/or formulate the eigenvalue equation by interpreting the associated integral operator as an impedance-type operator.

We present a new surface integral equation-based TCM formulation that is free of spurious modes for lossless and lossy dielectric and magneto-dielectric bodies without requiring any additional postprocessing techniques [11].

\section{FORMULATION}

To formulate the new TCM formulation, let us start with the EFIE (without the excitation) written in the exterior $(j=1)$

$$
\eta_{1} \boldsymbol{T}_{1}[\boldsymbol{J}]-\left(\boldsymbol{K}_{1}+\frac{1}{2} \boldsymbol{n} \times\right)[\boldsymbol{M}]=0
$$

and the MFIE written in the interior $(j=2)$

$$
\frac{1}{\eta_{2}} \boldsymbol{T}_{2}[\boldsymbol{M}]+\left(\boldsymbol{K}_{2}-\frac{1}{2} \boldsymbol{n} \times\right)[\boldsymbol{J}]=0 .
$$

Here $\boldsymbol{T}_{j}$ and $\boldsymbol{K}_{j}$ are the tangential EFIO and MFIO (without the residue term) of region $j$. Next eliminate $M$ from the equations by expressing it in terms of $\boldsymbol{J}$

$$
\boldsymbol{M}=\eta_{2}\left(\boldsymbol{T}_{2}\right)\left(\boldsymbol{K}_{2}+\frac{1}{2} \boldsymbol{n} \times\right)[\boldsymbol{J}]=\boldsymbol{P}[\boldsymbol{J}] .
$$

By substituting this into (11), gives an integral equation expressed solely in terms of the electric current

$$
\left(\eta_{1} \boldsymbol{T}_{1}-\left(\boldsymbol{K}_{1}+\frac{1}{2} \boldsymbol{n} \times\right) \boldsymbol{P}\right)[\boldsymbol{J}]=0 .
$$

This equation is similar to the generalized impedance boundary condition formulation for conducting bodies [6].

We note that equation (4) contains two parts, one related to the electric field radiated by an electric current, operator $\boldsymbol{Z}^{J}=\eta_{1} \boldsymbol{T}_{1}$, and the other one related to the field radiated by the magnetic current, operator $\boldsymbol{Z}^{M}=-\left(\boldsymbol{K}_{1}+1 / 2 \boldsymbol{n} \times\right)$. To relate the eigenvalues to the radiated power, the right hand side of the eigenvalue equation should contain the real part of $\boldsymbol{Z}^{J}$ and the imaginary part of $\boldsymbol{Z}^{M}$, multiplied with $i \boldsymbol{P}$. Consequently, the TCM eigenvalue equation for (4) reads [11]

$$
\left(\boldsymbol{Z}^{J}+\boldsymbol{Z}^{M} \boldsymbol{P}\right)\left[\boldsymbol{J}_{n}\right]=\left(1-i \lambda_{n}\right)\left(\boldsymbol{R}^{J}+i \boldsymbol{X}^{M} \boldsymbol{P}\right)\left[\boldsymbol{J}_{n}\right] .
$$

To avoid also the internal resonances associated with the EFIEtype formulation, a combined formulation is needed [11].

\section{NUMERICAL RESULTS}

For numerical solutions the surfaces are discretized with planar triangular elements and the equations are discretized with Galerkin's method and RWG basis and test functions.

Consider first a lossless dielectric sphere with radius $4.6 \mathrm{~mm}$ and $\varepsilon_{r}=40, \mu_{r}=1$. Fig. 1 shows the magnitudes of the eigenvalues of the three lowest order TE and TM modes computed with (5) and with analytical formulas [11].

The next example, Figs. 22 and 3. shows the real and imaginary parts of the eigenvalues for a lossy dielectric sphere with radius $4.6 \mathrm{~mm}$ and $\varepsilon_{r}=40+2 i, \mu_{r}=1$. The last example in Figure 4 shows the eigenvalues for a magneto-dielectric sphere with $r=4.6 \mathrm{~mm}$ and $\varepsilon_{r}=40, \mu_{r}=3.5$. 


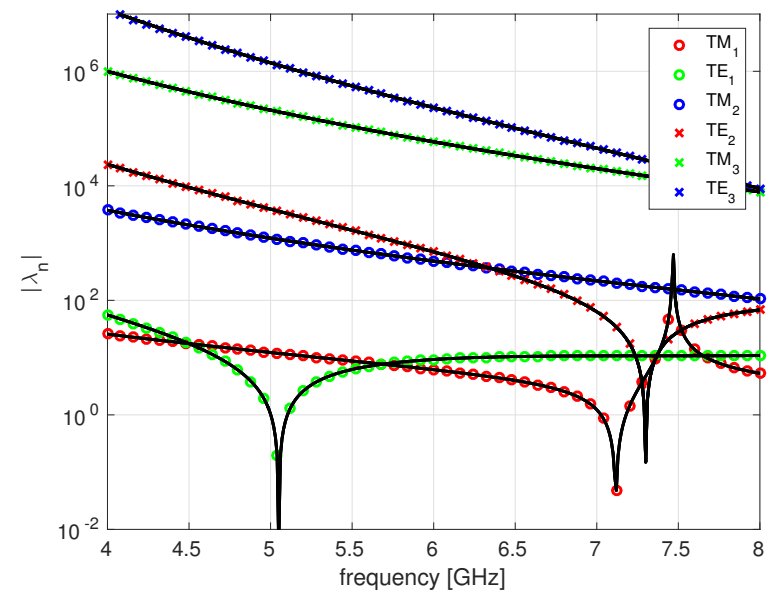

Fig. 1. Magnitudes of the eigenvalues for a lossless dielectric sphere, radius 4.6mm, $\varepsilon_{r}=40, \mu_{r}=1$. Numerical results are plotted with solid lines and analytical ones with symbols.

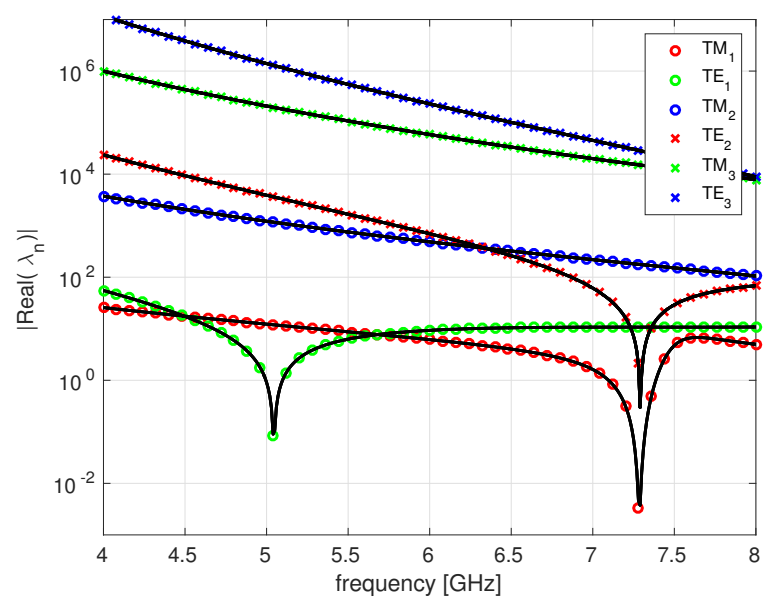

Fig. 2. Magnitudes of the real parts of the eigenvalues for a lossy dielectric sphere, radius $4.6 \mathrm{~mm}, \varepsilon_{r}=40+2 i, \mu_{r}=1$, similarly as in Fig. 1

\section{CONCLUSION}

A new spurious-free TCM formulation for lossless and lossy penetrable bodies is presented. In the formulation, the internal equations are only used to eliminate one of the currents from the equations and the required eigenvalue equation is formulated so that the obtained eigenvalues are related to the radiated power and have clear physical interpretation [11]. To avoid also the internal resonances associated with the EFIEtype formulation, a combined formulation is needed [11].

\section{REFERENCES}

[1] R.J. Garbacz and R.H. Turpin, "A generalized expansion for radiated and scattered fields", IEEE Trans. Antennas Propag., vol. AP-19, no. 3, pp. 348-358, 1971.

[2] R.F. Harrington and J.R. Mautz, "Theory of characteristic modes for conducting bodies", IEEE Trans. Antennas Propag., vol. AP-19, no. 5, pp. 622-628, 1971.

[3] Y. Chang and R.F. Harrington, "A surface formulation for characteristic modes of material bodies", IEEE Trans. Antennas Propag., vol. AP-25, no. 6, pp. 789-795, 1977.

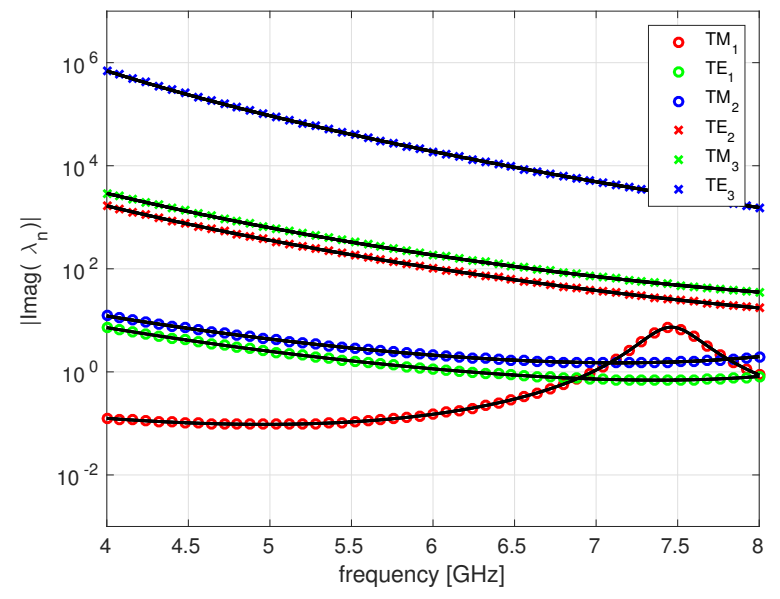

Fig. 3. Magnitudes of the imaginary parts of the eigenvalues for a lossy dielectric sphere, radius $4.6 \mathrm{~mm}, \varepsilon_{r}=40+2 i, \mu_{r}=1$, similarly as in Fig. 1

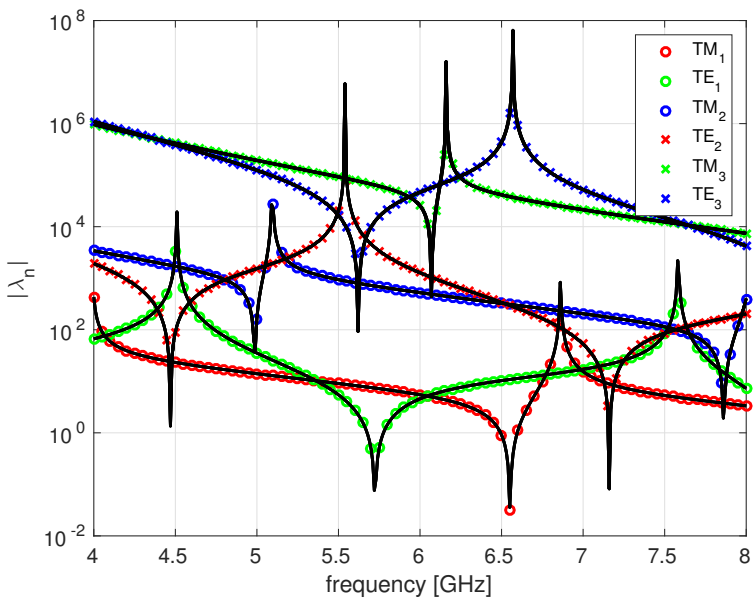

Fig. 4. Magnitudes of the eigenvalues for a lossless magneto-dielectric sphere, radius $4.6 \mathrm{~mm}, \varepsilon_{r}=40, \mu_{r}=3.5$, similarly as in Fig. 1]

[4] Z.T. Miers and B.K. Lau, "Computational analysis and verifications of characteristic modes in real materials", IEEE Trans. Antennas Propag., vol. 64, no. 7, pp. 2595-2607, July 2016.

[5] Q.I. Dai, Q.S. Liu, H.U.I. Gan, and W.C. Chew, "Combined field integral equation based theory of characteristic mode", IEEE Trans. Antennas Propag., vol. 63, no. 9, pp. 3973-3981, Sep. 2015.

[6] Z.G. Qian, W.C. Chew, and R. Suaya, "Generalized impedance boundary condition for conductor modeling with surface integral equations", IEEE Trans. Microw. Theory Techn., vol. 55, no. 11, pp. 2354-2364, Nov. 2007.

[7] Y. Chen and C.F. Wang, Characteristic modes - Theory and applications in antenna engineering, John Wiley \& Sons, New Jersey, 2015.

[8] Y. Chen, "Alternative surface integral equation-based characteristic mode analysis of dielectric resonator antennas", IET Mircow. Antennas \& Propag., vol. 10, no. 2, pp. 193-201, 2016.

[9] F.G. Hu and C.F. Wang, "Integral equation formulations for characteristic modes of dielectric and magnetic bodies", IEEE Trans. Antennas Propag., vol. 64, no. 11, pp. 4770-4776, 2016.

[10] R. Lian, J. Pan, and S. Huang, "Alternative surface integral equation formulations for characteristic modes of dielectric and magnetic bodies", IEEE Trans. Antennas Propag., vol. 65, no. 9, pp. 4706-4716, Sep. 2017.

[11] P. Ylä-Oijala, H. Wallén, D.C. Tzarouchis, and A. Sihvola, "Surface integral equation based characteristic mode formulation for penetrable bodies", submitted 